\title{
GÉNÉRALITÉS SUR LES DISPOSITIFS DE FRANCHISSEMENT
}

\author{
M. LARINIER \\ CSP-CEMAGREF, GHAAPPE - Institut de Mécanique des Fluides \\ Avenue du Professeur Camille Soula - 31400 TOULOUSE
}

\section{PRINCIPE DES PASSES A POISSONS}

Le principe général des dispositifs de franchissement consiste à attirer les migrateurs en un point déterminé du cours d'eau à l'aval de l'obstacle et à les inciter, voire à les obliger à passer en amont, en leur ouvrant une voie d'eau (passes à poissons stricto sensu) ou en les piégeant dans une cuve et en déversant celle-ci en amont (ascenseurs et systèmes de piégeage et de transport).

Pour qu'une passe puisse être considérée comme efficace, le poisson doit en trouver l'entrée, la franchir sans retard, stress ou blessures préjudiciables à sa migration vers l'amont.

La conception d'un dispositif de franchissement doit prendre en compte certains aspects du comportement des espèces migratrices. En particulier, son efficacité est fortement liée aux conditions hydrodynamiques et au respect de vitesses appropriées dans l'ouvrage.

Les vitesses doivent rester compatibles avec les capacités de nage des espèces concernées : les passes doivent permettre le passage de tous les individus et pas uniquement celui des "athlètes".

Certaines espèces sont très sensibles à certains régimes ou conditions d'écoulement : des chutes trop importantes, une aération et une turbulence excessives, l'existence de zones de recirculation trop vastes, des vitesses trop faibles peuvent être autant de causes de blocage pour le poisson.

Outre les facteurs hydrodynamiques, le poisson est sensible à d'autres paramètres environnementaux (teneur en oxygène dissous, température, bruit, odeur, etc...) qui peuvent s'avérer dissuasifs. C'est le cas en particulier lorsque la qualité de l'eau alimentant la passe se distingue de celle transitant par le barrage (faibles teneurs en oxygène dissous, différences de température...).

Le poisson possède aussi des exigences ou préférences en ce qui concerne l'intensité lumineuse ambiante. Des conditions de lumière à l'entrée et dans l'ouvrage de franchissement trop différentes de celles existant au pied de l'obstacle peuvent s'avérer dissuasives : gradient d'éclairement trop violent à l'entrée d'ouvrage, conditions d'éclairage insuffisantes dans la passe ou au contraire éclairage de la passe la nuit pour les espèces lucifuges.

L'influence de la plupart de ces paramètres sur le comportement des espèces migratrices est cependant mal documentée à l'heure actuelle et provient le plus souvent d'observations ponctuelles ; c'est pourquoi elle est pour l'ingénieur difficilement traduisible en termes de critères de dimensionnement.

\section{LES DIFFÉRENTS TYPES DE DISPOSITIFS DE FRANCHISSEMENT}

Le type de passe qui est le plus couramment utilisé est incontestablement la passe à bassins successifs ; elle consiste en une série de bassins partant du pied de l'obstacle et rejoignant le bief amont. Les cloisons séparant les bassins sont munis de déversoirs, d'orifices ou de fentes verticales par où transite le débit de la passe et qui contrôlent le 
niveau d'eau dans chaque bassin. Les bassins jouent un double rôle : ils assurent une dissipation convenable de l'énergie de l'eau transitant dans la passe tout en assurant des zones de repos pour le poisson. La dénivellation entre deux bassins successifs est fonction des espèces migratrices considérées. La pente d'une passe à bassins varie le plus souvent entre $10 \%$ et $15 \%$.

Dans les échelles à ralentisseurs - ou "Denil", du nom de leur inventeur - sont disposés sur le fond et/ou sur les parois d'un canal à forte pente (jusqu'à $20 \%$ ) des déflecteurs de formes plus ou moins complexes destinés à réduire les vitesses moyennes de l'écoulement en créant des courants hélicoïdaux.

L'écluse à poissons (ou écluse "Borland") fonctionne suivant un principe voisin de celui d'une écluse de navigation : les migrateurs sont piégés dans un sas puis éclusés comme le serait un bateau.

D'autres dispositifs consistent à piéger les migrateurs dans une cuve puis à les transférer à l'amont soit par ascenseur ou funiculaire, soit encore tout simplement par camion.

La passe de type "rivière artificielle" (parcours à canoë-kayak par exemple) consiste à relier biefs amont et aval par un chenal creusé dans l'une des rives, chenal dont le fond et les parois sont garnis de rugosités ou d'obstacles (épis, seuils...) reconstituant en quelque sorte un cours d'eau naturel. La pente d'un tel ouvrage ne peut cependant dépasser quelques pour cent ; son implantation, du fait de sa longueur, se révèle souvent problématique. II s'intègre par contre très bien dans le paysage.

Il existe de nombreuses autres solutions atypiques qui consistent à aménager plus ou moins sommairement un obstacle pour en faciliter le franchissement par les migrateurs ; ces solutions restent cependant limitées aux obstacles de hauteur modérée. On citera les prébarrages, formés de plusieurs murets ou seuils installés à l'aval de l'obstacle créant des grands bassins fractionnant la chute. Ces prébarrages peuvent être installés sur une fraction importante, voire même sur la totalité de la largeur du cours d'eau en aval de l'obstacle. Quelquefois une simple brèche, dans le cas d'un barrage à parement aval vertical, une écharpe ou une goulotte, dans le cas d'un seuil à parement aval incliné, se révèlent suffisantes pour assurer le passage du poisson.

Une dernière solution - aussi efficace que radicale - consiste à supprimer l'obstacle : ce peut être possible, dans le cas par exemple d'une ancienne chaussée de moulin qui n'est plus exploitée. Cette solution présente parallèlement l'avantage de recréer dans la zone d'influence amont de la chaussée des secteurs courants propices à la reproduction des adultes ou à la croissance des juvéniles.

\section{CRITÈRES DE CHOIX D'UN DISPOSITIF DE FRANCHISSEMENT}

II n'existe pas un type de passe "miracle" plus efficace que tous les autres: l'expérience montre que de nombreuses passes à bassins, à ralentisseurs, de même que des ascenseurs se sont également révélés efficaces - ou inefficaces.

II serait tentant de ranger les différents obstacles en plusieurs classes - suivant leur hauteur par exemple - et de proposer pour chacune d'elles et en fonction des espèces migratrices un type de passe approprié.

La multiplicité des contraintes et des facteurs (d'ordre biologique, hydrologique, hydraulique, topographique...) fait que chaque situation est un cas d'espèce et l'expérience montre qu'une telle classification rigide est susceptible au contraire d'entraîner de grossières erreurs ou des coûts prohibitifs.

Les quelques points suivants aideront à déterminer pour une situation donnée le type de passe le plus approprié.

1. Lorsque l'on a affaire à plusieurs espèces migratrices (saumons, truites de mer, truites, poissons blancs, etc...), la passe à bassins successifs est généralement la meilleure solution, beaucoup moins sélective que les passes à ralentisseurs. On dimensionnera la passe pour l'espèce la plus exigeante (en termes de chute entre bassins, de vitesses, de puissance dissipée volumique...). 
Les passes à bassins à échancrures déversantes, associées ou non à des orifices de fond, sont les plus intéressantes car elles s'adaptent à de nombreux cas de figure : débit disponible de quelques dizaines de litres à plusieurs $\mathrm{m}^{3} / \mathrm{s}$ et variations de niveau amont significatives sans nécessiter l'installation d'une section de régulation à l'amont (vannes automatiques, orifices noyés etc...), à condition que les échancrures soient suffisamment profondes.

Les passes à fentes verticales sont particulièrement adaptées aux sites présentant de grandes fluctuations des niveaux d'eau amont et/ou aval. Pour les grands migrateurs, compte tenu de la largeur minimale de la fente à donner, elles nécessitent cependant des débits importants (supérieurs à $0.75 \mathrm{~m}^{3} / \mathrm{s}$ environ).

De façon générale, les passes à orifices noyés présentent peu d'intérêt, sauf en tant que section de régulation en tête d'un dispositif de franchissement, dans le cas de variation significative du niveau d'eau amont. Elles présentent par ailleurs l'inconvénient de piéger les corps flottants (bois, bouteilles, polystyrène, etc...) qui viennent s'accumuler en surface dans les bassins sans pouvoir être évacués.

Dans le cas d'un cours d'eau à transport solide notable, on évitera l'installation de bassins trop profonds susceptibles de pièger les sédiments grossiers et on fera en sorte que les communications entre les bassins se fassent jusqu'au radier pour faciliter l'évacuation des matériaux.

L'alose pose un problème particulier pour la conception et le dimensionnement des passes à bassins : cette espèce est beaucoup plus sensible que les salmonidés aux conditions hydrodynamiques et des précautions particulières doivent être prises pour assurer son passage. De bons résultats ont été obtenus aux USA sur l'alose américaine (alosa sapidissima) et en France sur la grande alose (alosa alosa) en respectant un certain nombre de conditions : niveau de turbulence faible dans les bassins, échancrures ou fentes de largeur suffisante $(>0.45 \mathrm{~m})$ situées le long des parois, écoulements de surface évitant tout jet plongeant, limitation des zones de recirculation. Les passes à une ou deux fentes verticales se révèlent efficaces, en dépit d'un comportement d'aller et retour du poisson dans les bassins qui semble inhérent à l'espèce.

2. Les passes à ralentisseurs sont relativement sélectives et ne sont adaptées qu'aux espèces possédant des capacités de nage suffisantes en termes de vitesse de nage et d'endurance (grands salmonidés migrateurs, lamproies, grands cyprinidés d'eau vive). Elles ne sont pas adaptées aux poissons d'une longueur inférieure à une trentaine de $\mathrm{cm}$. Bien que des aloses aient été contrôlées dans plusieurs passes à ralentisseurs, il a été observé que cette espèce n'est pas à l'aise pour négocier les courants hélicoïdaux caractérisant l'écoulement dans ce type de passe et qu'elle s'y engage difficilement, sans doute à cause de l'aération et de la turbulence de l'écoulement.

Les passes à ralentisseurs sont particulièrement adaptées aux cours d'eau de petite ou moyenne importance sur lesquels le débit disponible pour le dispositif de franchissement n'est que de quelques centaines de litres/seconde et aux anciens seuils ou chaussées de moulins de faible hauteur à parement aval incliné dans lesquels elles s'intègrent facilement.

L'installation de ce type de passe perd généralement de son intérêt lorsque la hauteur de l'obstacle à franchir devient plus importante : la nécessité de prévoir des bassins de repos (tous les deux mètres de chute environ) augmente la longueur du dispositif et peut rendre tout aussi intéressante l'installation d'une passe à bassins successifs.

Certains types de passes à ralentisseurs (ralentisseurs de fond en chevrons épais) peuvent être utilisés comme glissières à canoë-kayak dans la mesure évidemment où on leur donne une largeur suffisante (1.40 m minimum).

On évitera l'installation de passes à ralentisseurs dans les cours d'eau transportant des matériaux grossiers (gros galets, blocs) qui risquent de venir se déposer et se coincer entre les ralentisseurs, réduisant sinon annulant l'efficacité de ceux-ci. Par contre les courants hélicoïdaux générés dans ce type de passe sont capables d'évacuer sans problème les limons, sables et graviers. 
3. Pour les ouvrages de hauteur importante, l'installation d'un ascenseur ou d'une écluse Borland est plus facile et généralement moins coûteuse que celle d'une passe classique. L'écluse à poissons présente l'inconvénient majeur de posséder un fonctionnement discontinu; on lui préfère généralement l'ascenseur dont la technologie a progressé ces dernières années en France. Par contre, la solution d'une écluse peut s'avérer intéressante pour un obstacle de hauteur modérée, lorsque la configuration de celui-ci exclut l'installation d'une passe classique.

L'efficacité d'un ascenseur est fortement liée à la porosité de ses grilles : son utilisation devient problématique pour les petites espèces qui exigent des grilles à espacements très réduits, d'un entretien trop astreignant.

La relative complexité du fonctionnement de ces deux types d'ouvrage (présence de plusieurs organes mobiles - vannes, cuves, grilles - automatisés) se traduit par des pannes ou des périodes de dysfonctionnement dont la fréquence et la durée peuvent s'avérer non négligeables. Les coûts de maintenance sont par ailleurs plus élevés que ceux afférents aux passes classiques. C'est la raison pour laquelle on peut leur préférer dans certains cas des passes "statiques" (sans aucun organe mobile) d'un génie civil plus coûteux mais d'un entretien moins lourd et d'un fonctionnement plus fiable.

\section{PRINCIPALES CAUSES DE MAUVAIS FONCTIONNEMENT DES PASSES A POISSONS}

Lorqu'on analyse l'origine du dysfonctionnement des dispositifs de franchissement reconnus comme peu ou pas efficaces, un certain nombre de causes sont fréquemment évoquées:

- le manque d'attractivité de l'ouvrage, résultant soit d'une mauvaise localisation de l'ouvrage, soit d'un débit insuffisant, peu en rapport avec le débit du cours d'eau,

- un mauvais calage de l'ouvrage eu égard aux variations des niveaux d'eau amont et aval en période de migration, se traduisant par une sous ou une suralimentation de l'ouvrage, ou encore par une chute trop forte à l'entrée. Cela peut provenir soit d'une méconnaissance des conditions de niveaux lors de l'élaboration du projet, soit d'une évolution ultérieure de ces niveaux (gestion différente de l'ouvrage, abaissement du fil d'eau à l'aval...),

- un mauvais dimensionnement de l'ouvrage : bassins de volumes trop réduits se traduisant par une aération et des turbulences excessives, chutes entre bassins trop importantes, profondeurs d'eau insuffisantes pour le poisson, hydrodynamique des bassins non adaptée aux espèces migratrices,

- un colmatage ou un encombrement fréquent de l'ouvrage résultant soit d'une mauvaise protection contre les corps dérivants, soit d'une implantation trop exposée, soit tout simplement d'un défaut d'entretien de la part du propriétaire de l'ouvrage. L'encombrement par les corps dérivants peut se traduire par une mauvaise alimentation en eau de l'ouvrage (colmatage des pertuis, des grilles d'injection de débit d'attrait complémentaire ...) et par une entrave au passage des poissons. A ce titre, les passes à ralentisseurs et les passes à bassins comportant des orifices noyés sont particulièrement vulnérables. Des obstructions même très partielles peuvent rendre le passage des poissons impossible sans pour autant induire de perturbations très visibles de l'écoulement,

- un mauvais fonctionnement des organes assurant la régulation du débit et des chutes de certains ouvrages (vannes asservies ...) ou assurant le fonctionnement de l'ouvrage dans le cas des ascenseurs et écluses (vannes, treuils de manoeuvre des grilles, de la cuve, ...).

Des causes externes à la passe elle-même peuvent également intervenir sur le fonctionnement de celle-ci. Il s'agit le plus souvent de l'altération de l'attractivité de la passe consécutive à des manoeuvres de vannes, à une gestion des turbines ou plus généralement à des conditions d'écoulement au pied de l'obstacle non prises en compte lors de la conception du projet ; cela peut se traduire par la perturbation de l'écoulement à l'entrée de la passe ou par l'attraction et le piégeage du poisson dans une zone relativement éloignée de celle-ci. 


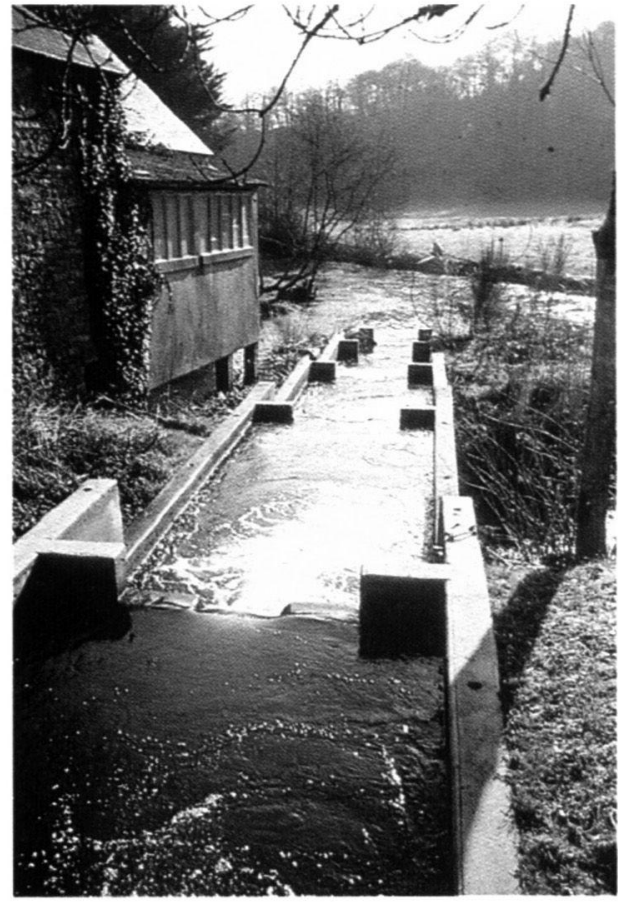

Photo 1 : Passe à bassins successifs sur le Steïr (Finistère).

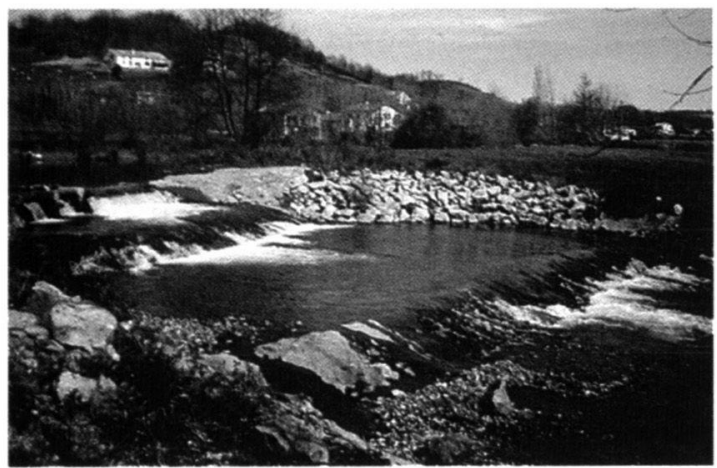

Photo 3 : Prébarrage en enrochements jointoyés au bitume de Zaldubia sur la Nivelle (Pyrénées-Atlantiques).

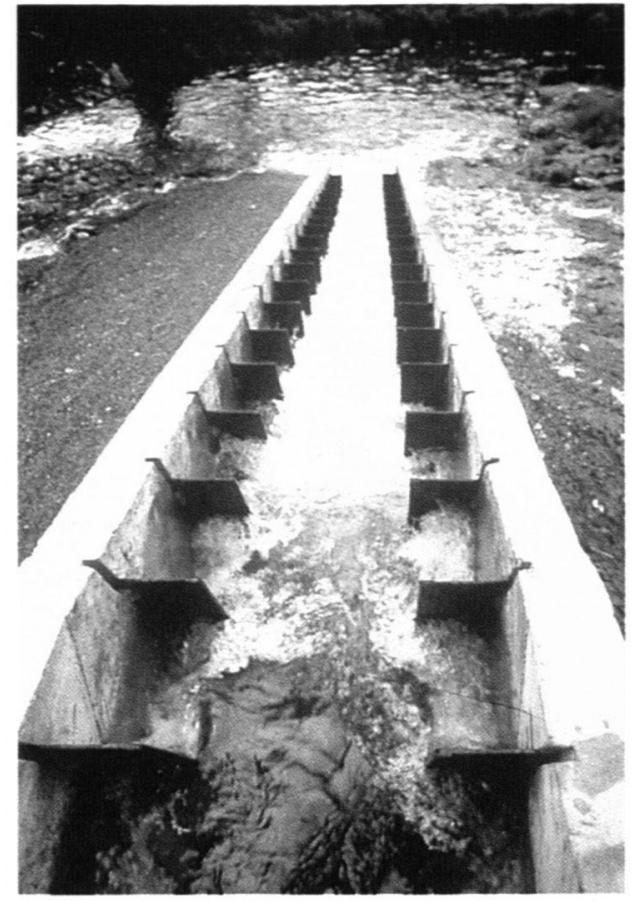

Photo 2 : Passe à ralentisseurs plans de Quinipily sur l'Evel (Morbihan).

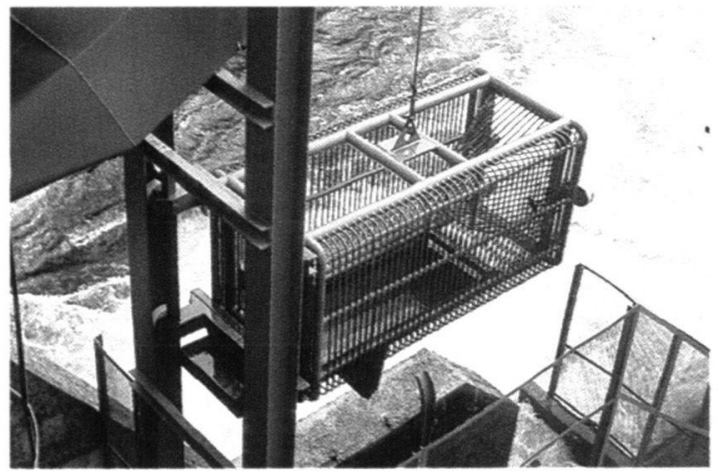

Photo 4 : Ascenseur à poissons sur le Loc'h (Morbihan).

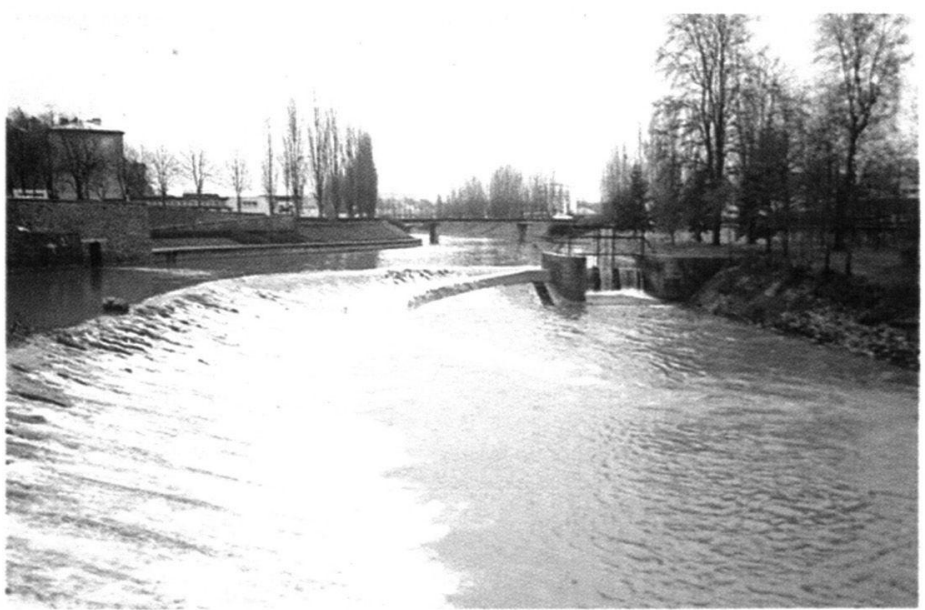

Photo 5 : Passe en écharpe sur la Vire à St-Ló (Manche). 